\title{
ЭКОНОМИЧЕСКАЯ ДЙНАМИКК В ЛЕОНТЬЕВСКОЙ МОДЕЛИ С КОМПЕНСАЦИЕЙ ОСТАТОЧНЫХ УЩЕРБОВ
}

\author{
(Представил Г. Вайникко)
}

\section{1. Постановка задачи и основной результат}

Традиционный экономический механизм нацелен на расширенное воспроизводство с максимально возможными темпами. Процесс расширенного воспроизводства можно описать с помощью простейшей динамической модели Неймана-Леонтьева:

$$
x_{t} \geqslant A x_{t}+B x_{t+1},
$$

где $\dot{A}$ и $B$ - соответственно матрицы коэффициентов текущих затрат и затрат на накопление; вектор валовой продукции $x_{t}$, таким образом, распределяется на текущее производственное потребление и накопление. Этой модели соответствует понятие максимального сбалансированного роста - его темп и межотраслевые пропорции определяются решением задачи $\max \{\alpha \mid x \geqslant A x+\alpha B x, x \geqslant 0, x \neq 0\}$. Известно, чтоं для динамических задач планирования, формулируемых на основе модели (1), оптимальные траектории при достаточно больших горизонтах планирования близки к магистрали - траектории максимального сбалансированного роста.

Концепции максимального роста (накоплсния) соответствует убывающее предпочтение благ во времени, т. е. будущие блага ценятся ниже, чем сегодняшние. В случае долгосрочных проектов это прриводит к тому, что запасы ресурсов, предназначенные к эксплуатации в отдаленном будущем, получают ничтожную оценку. Очевидно, что недооценка последствий хозяйственной деятельности оборачивается в будущем дополнительными издержками. Однако эти последствия никак не отражаются в балансе затрат в момент нанесения ущерба ресурсам илй окружающей среде, т. е. в экономическом механизме отсутствует обратная связь,

В [ $\left.{ }^{1}\right]$ предложено следующее обобщение модели (1) с целью учета отрицательных последствий хозяйственной деятельности. Баланс распределения валовой продукции года $t$

$$
x_{t} \geqslant A x_{t}+B x_{t+1}+D x_{t-1}
$$

в отличие от (1) включает также затраты $D x_{t-1}$, вызванные пронзводством в предыдущий период (в частности, на компенсащию остаточного ущерба); здесь $D$ - матрица соответствующих коэффициентов.

Как и для модели (1), здесь также может быть поставлена задача нахождения் сбалансированных решений:

$$
x \geqslant A x+\alpha B x+\frac{1}{\alpha} D x, \quad x \geqslant 0, x \neq 0,
$$

однако в данном случае оказывается, что эта задача имеет как максимальное, так и минимальное решенне, 
Пусть далее везде выполняется

Предположение. Квадратные матрицы $A=\left(a_{i j}\right), \quad B=\left(b_{i j}\right)$, $D=\left(d_{i j}\right)$ состоят из неотрицательных элементов, матрица $A$ неразложима и выполняется следующее условие продуктивности:

$$
\sum_{j=1}^{n} a_{i j}+\sum_{j=1}^{n} b_{i j}+\sum_{j=1}^{n} d_{i j}<1
$$

для $i=1, \ldots, n$.

Тогда, как известно, матрица $(I-A)^{-1}$ существует и поэлементно положительна. Обозначим $\hat{B}=(I-A)^{-1} B, \hat{D}=(I-A)^{-1} D . \quad$ Справедливы следующие утверждения *.

Л ем м а 1. Существуют, единственны и полояительны скаляр $\alpha^{*}$ и нормированный вектор $x^{*}$, доставляющие решение задаче

$$
\max \left\{\alpha \mid x \geqslant\left(\alpha \hat{B}+\frac{1}{\alpha} \hat{D}\right) x, x \geqslant 0, x \neq 0\right\}
$$

причем $x^{*}-$ собственный вектор матрицы $\alpha^{*} \hat{B}+\frac{1}{\alpha^{*}} \hat{D}$, отвечающций ее максимальному собственному числу 1.

Л е м м а 2. Существуют, единственны и положительны скаляр $\alpha_{\text {н }}$ и нормированный вектор $x_{*}$, доставляющце решение задаче

$$
\min \left\{\alpha \mid x \geqslant\left(\alpha \hat{B}+\frac{1}{\alpha} \hat{D}\right) x, x \geqslant 0, x \neq 0\right\},
$$

причем $x_{*}-$ собственный вектор матрицы $\alpha_{*} \hat{B}+\frac{1}{\alpha_{*}} \hat{D}$, отвечающий ее максимальному собственному числу 1.

Таким образом, в отличие от традиционной модели (1) здесь наряду с максимальным темпом расширения производства $\alpha^{*}$ возникает и минимальный темп $\alpha_{*}$ убывания остаточных ущербов. Нетрудно убедиться, что при выполнении условия (3) имеет место $\alpha^{*}>1>\alpha_{*}$.

Рассмотрим следующую задачу планирования на интервале времени $t=1, \ldots, T$, основанную на модели (2).

$$
\begin{aligned}
& c_{1} x_{1}+c_{T} x_{T} \rightarrow \min , \\
& x_{t} \geqslant 0, \quad t=1, \ldots, T, \\
& x_{1} \geqslant A x_{1}+B x_{2}+d_{1}, \\
& x_{t} \geqslant A x_{t}+B x_{t+1}+D x_{i-1}, \quad t=2, \ldots, T-1, \\
& x_{T} \geqslant A x_{T}+D x_{T-1}+r_{T},
\end{aligned}
$$

где заданы неотрицательные $n$-мерные векторы $d_{1}, r_{T}, c_{1}, c_{T}$ и квадратные матрицы $A, B, D$. Искомой является последовательность векторов валовой продукции $x_{t}$, удовлетворяющая балансовым ограничениям и обеспечивающая производство заданных объемов конечной продукции в начале и конце планового периода при минимальных суммарных затратах экзогенного ресурса. Векторы конечной продукции $d_{1}$ и $r_{T}$ можно интерпретировать соответственно как унаследованные от допланового периода ущербы и переходящие на послеплановый период ресурсы.

Основной результат работы представляет собой своего рода теорему о магистрали.

* Формулируемые здесь и далее леммы приводятся без доказательств. 
Т еорем а. Для любого $\varepsilon>0$ существует целое $T(\varepsilon)$ такое, что если $T>2 T(\varepsilon)$, то для всякого оптимального решения $\left\{\bar{x}_{t}\right\}_{t=1}^{T}$ задачи (4) имеет место разложение вида $\bar{x}_{t}=x_{t}^{\prime}+x_{t}^{\prime \prime}$ такое, что выполняется

$$
\begin{aligned}
& \left\|\frac{x_{t}^{\prime}}{\left\|x_{t}^{\prime}\right\|}-x^{*}\right\|<\varepsilon, \quad\left|\frac{\left\|x_{t+1}^{\prime}\right\|}{\left\|x_{t}^{\prime}\right\|}-\alpha^{*}\right|<\varepsilon, \\
& \left\|\frac{x_{t}^{\prime \prime}}{\left\|x_{t}^{\prime \prime}\right\|}-x_{*}\right\|<\varepsilon, \quad\left|\frac{\left\|x_{t+1}^{\prime \prime}\right\|}{\left\|x_{t}^{\prime \prime}\right\|}-\alpha_{*}\right|<\varepsilon
\end{aligned}
$$

nрu $T(\varepsilon) \leqslant t \leqslant T-T(\varepsilon)$.

Этот результат показывает на примере простейшей модели, что учет в составе затрат отрицательных последствий производственной деятельности существенно меняет картину оптимального экономического роста. Он оказывается результатом взаимодействия двух составляющих, которые отвечают противоположным целям: максимальному росту производства (накоплению) и наискорейшему убыванию остаточных ущербов, вызванных хозяйственной деятельностью в прошлом. Қаждой из составляющих отвечает своя магистраль. Составляющая накопления $\left\{x_{t}^{\prime}\right\}$, согласно теореме, близка к траектории сбалансированного роста с темпом $\alpha^{*}$, а составляющая компенсации $\left\{x_{t}{ }^{\prime \prime}\right\}-$ к траектории сбалансированного сокращения производства с темпом $\alpha_{\star}$. Соотношение между двумя составляющими определяется матрицами $B$ и $D$, а также краевыми условиями $d_{1}$ и $r_{T}$. В частности, если вектор $d_{1}$ ущербов, унаследованных от допланового периода, достаточно велик (относитсльно вектора $r_{T}$ ), то результирующая траектория $\left\{x_{t}\right\}$ может характеризоваться сөкращением объемов производства. Аналогичный данной теореме факт можно доказать для задачи, двойственной к (4). В частности, в случае, когда вектор коэффициентов $c_{T}$ достаточно велик по сравнению с $c_{1}$, для оптимального решения характерен рост переменных, т. е. двойственных оценок задачи (4).

\section{2. Вспомогательные результаты}

Заметим, что в случае $d_{1}=r_{T}=0$ задача (4) имеет тривиальное решение $x_{t}=0$ для $t=1, \ldots, T$, так что естественно считать, что хотя бы один из векторов $d_{1}$ и $r_{T}$ ненулевой. Обозначим $\hat{d}_{1}=(I-A)^{-1} d_{1}$, $\hat{r}_{T}=(I-A)^{-1} r_{T}$. Допустимые решения задачи (4) удовлетворяют неравенствам

$$
\begin{aligned}
x_{t} & \geqslant 0, \quad t=1, \ldots, T, \\
x_{1} & \geqslant \hat{B} x_{2}+\hat{d}_{1}, \\
x_{t} & \geqslant \hat{B} x_{t+1}+\hat{D} x_{t-1}, \quad t=2, \ldots, T-1, \\
x_{T} & \geqslant \hat{D} x_{T-1}+\hat{r}_{T},
\end{aligned}
$$

которые получаются путем домножения на положительную матрицу $(I-A)^{-1}$ обеих частей соответствующих неравенств из (4). В силу строгой положительности матриц $\hat{B}$ и $\hat{D}$ в любом решении системы (5) все векторы строго положительны. В частности, положительны все векторы в оптимальном решении $\left\{\bar{x}_{t}\right\}_{t=1}^{T}$ задачи (4). Исходя из этого факта и условий допслняющей нежесткости в двойственных задачах 
линейного программирования, нетрудно убедиться, ӵто оптимал̆ьные решения в задаче, двойственной к (4) также положительны. Тем самым в (4) для оптимального решения $\left\{\bar{x}_{t}\right\}_{t=1}^{T}$ все ограничения выполняются как равенства. Тогда, в силу линейности всех соотношений в (4), можно записать $\bar{x}_{t}=x_{t}^{\prime}+x_{t}^{\prime \prime}$ для $t=1, \ldots, T$, где $\left\{x_{t}^{\prime}\right\}-$ peшіение системы

$$
\begin{aligned}
x_{t} & \geqslant 0, \quad t=1, \ldots, T, \\
x_{1} & =\hat{B} x_{2}, \\
x_{t} & =\hat{B} x_{t+1}+\hat{D} x_{t-1}, \quad t=2, \ldots, T-1, \\
x_{T} & =\hat{D} x_{T-1}+\hat{r}_{T},
\end{aligned}
$$

a $\left\{x_{t}^{\prime \prime}\right\}$ - решение системы

$$
\begin{aligned}
x_{t} & \geqslant 0, \quad t=1, \ldots, T, \\
x_{1} & =\hat{B} x_{2}+\hat{d}_{1}, \\
x_{t} & =\hat{B} x_{t+1}+\hat{D} x_{t-1}, \quad t=2, \ldots, T-1, \\
x_{T} & =\hat{D} x_{T-1} .
\end{aligned}
$$

Рассмотрим отдельно систему (6) и будем излагать основные этапы доказательства утверждения теоремы, относящегося к составляющей $\left\{x_{t}^{\prime}\right\}$. Заметим, что если в системе (7) изменить направление рекурсий, то она по форме совпадет с (6). Поэтому результаты, получаемые при исследовании решений системы (6), применимы с соответствующими йзменениями к решениям (7), т. е. к составляющей $\left\{x_{t}^{\prime \prime}\right\}$.

Итак, задача свелась к исследованию неотрицательных решений системы разностных уравнений второго порядка

$$
\hat{B} x_{t+1}-x_{t}+\hat{D} x_{t-1}=0 \text {, }
$$

где $t=2, \therefore ., T-1$.

Введем обозначения: $2 n$-мерные векторы $z_{t}=\left(x_{2 t+1}, x_{2 t+2}\right)$ и матрицы размерности $2 n \times 2 n$

$$
\mathfrak{B}=\left(\begin{array}{rr}
\hat{B} & 0 \\
-I & \hat{B}
\end{array}\right), \quad \mathfrak{D}=\left(\begin{array}{rr}
-\hat{D} & I \\
0 & -\hat{D}
\end{array}\right) .
$$

Т̃ огда систему (8) можно представить в виде

$$
\mathfrak{B} z_{t}=\mathfrak{D} z_{t-1},
$$

где $t=1, \ldots,[T / 2]-1$, т. е. перейти к системе разностных уравнений первого порядка за счет увеличения размерности. При этом, однако, матрицы $\mathfrak{B}$ и $\mathfrak{D}$ содержат отрицательные элементы и, вообще говоря, необратимы, что исключает традиционные подходы к исследованию асимптотического вида решений, которые основаны как раз на свойствах неотрицательных матриц.

В этой работе будут использованы результаты о решениях систем вида (9), полученные для достаточно общего случая в $\left[{ }^{2,3}\right]$.

О пр едел ен и е. Последовательность $\left\{z_{t}\right\}_{t=0}^{N}$, удовлетворяющцая (9) при $t=1, \ldots, N$, называется $N$-цепочкой, в частности, если $N=\infty$, - бесконечной цепочкой. Вектор $z_{0}$, являющцйся началом $N$-цепочки, называется $N$ - продолжимым.

Обозначим через $R_{N}$ множество $N$-продолжимых вскторов. Очевидно, что $R_{N}$ - линейное подпространство, причем $R_{1} \supseteq R_{2} \equiv \ldots \supseteq R_{\infty}$. 
Если $(\mathfrak{B}, \mathfrak{D})$ - регулярный пучок матриц, т. е. найдется чйсло $\lambda_{0}$ такое, что $\operatorname{det}\left(\lambda_{0} \mathfrak{B}-\mathfrak{D}\right) \neq 0$ (нетрудно убедиться, чито для определенных выше матриц $\mathfrak{B}$ и $\mathfrak{D}$ это условие выполнено), то справедливы следующие утверждения.

Л е м м а $3\left[{ }^{2,3}\right] . R_{\infty}=R_{2 n}$, т. е. всякий $N$-продолжимый вектор, где $N \geqslant 2 n$, является бесконечно продолжимым.

Л е м м а $4\left[{ }^{3}\right]$. Если $\left\{z_{t}\right\}_{0}{ }^{N}-N$-цегіочка и $N>2 n$, то найдется матрица $G$ такая, что $z_{t+1}=G z_{t}$ для $t=1, \ldots ; N-2 n$.

Из леммы 4 следует, что для изучения асимптотики решений системы (9) достаточно исследовать спектр матрицы $G$, однако о его связи со спектром пучка матриц $(\mathfrak{B}, \mathfrak{D})$ известно лишь следующее.

Лемм а $5\left[{ }^{3}\right]$. Если $z \in R_{\infty}$, то для числа $\mu$ выполняется $G z=\mu z$ тогда и только тогда, когда $(\mu \mathfrak{B}-\mathfrak{D}) z=0$.

Число $\mu$ и вектор $z$ (вообще говоря, комплексные), для которых выполняется равенство $(\mu \mathfrak{B}-D) z=0$, будем называть соответственно собственным числом и собственным вектором пучка матриц (B, D).

Используя лемму 1, можно доказать следующие утверждения о спектре пучка $(\mathfrak{B}, \mathfrak{D})$.

Л ем м а 6 (i). $\left(\alpha^{*}\right)^{2}$ - максимальное среди вещцественных собственных чисел пучка (ㄹ, D), ему соответствует единственный, с точностью до умножения на скаляр, собственный вектор $z^{*}=\left(x^{*}, \alpha^{*} x^{*}\right)$.

(ii). Пусть $\lambda$ - собственное число пучка (ㄱ, D) такое, что $|\lambda|=$ $=\left(\alpha^{*}\right)^{2}$, тогда $\lambda=\left(\alpha^{*}\right)^{2}$.

Перейдем к исследованию решения $\left\{x_{t}^{\prime}\right\}$ системы (8). Положим $z_{t}=\left(x_{2 t+1}^{\prime}, x_{2 t+2}^{\prime}\right)$ для $t=0, \ldots$, [T/2]-1, так что в силлу (9) и леммы $4 \quad z_{t}=G^{t} z_{0}$ для $t=0, \ldots$, [T/2]-n-1.

Пусть $\lambda_{1}, \ldots, \lambda_{m}-$ собственные числа оператора $G ; e_{k \theta}$, где $\theta=1, \ldots, \theta_{k}$, - система нормированных корневых векторов в $C^{2 n}$, отвечающих собственному числу $\lambda_{k}$ (в частности, $e_{h 1}-$ его собственный вектор). Поскольку система всех корневых векторов линейного оператора $G: R^{2 n} \rightarrow R^{2 n}$ образует базис в $C^{2 n}$, то найдутся вещественные числа $v_{k \theta}$, где $\theta=1, \ldots, \theta_{k} ; k=1, \ldots, m$ такие, что

$$
z_{0}=\sum_{k=1}^{m} \sum_{\theta=1}^{\theta_{k}} v_{k \theta} e_{k \theta}
$$

Используя соотношения для корневых векторов:

$$
G e_{k 1}=\lambda_{k} e_{k 1}, \quad G e_{k \theta}=\lambda_{k} e_{k \theta}+e_{k \theta-1} \quad \text { для } \theta=2, \ldots, \theta_{k}
$$

(см. $\left[{ }^{4}\right]$, с. 188$)$, нетрудно вывести формулу

$$
G^{t} e_{k \theta}=\lambda_{k}^{t}\left(\frac{r_{\theta-1}(t)}{\lambda_{k}^{\theta-1}} e_{k 1}+\frac{r_{\theta-2}(t)}{\lambda_{k}^{\theta-2}} e_{k 2}+\ldots+r_{0}(t) e_{k \theta}\right)
$$

где $r_{j}(t)=\frac{1}{j !} t(t-1) \ldots(t-j+1) \quad$ - многочлен порядка $j, r_{0}(t) \equiv 1$.

Отсюда в силу $(10)$, когда $t$ достаточно велико (скажем, пз меньше нексторого $T_{0}$ ), можно записать

$$
z_{t}=G^{t} z_{0}=\sum_{k=1}^{m} \sum_{\theta=1}^{\theta_{k}} v_{k \theta} \lambda_{k}^{t-\theta+1} \frac{t^{\theta-1}}{(\theta-1) !}\left(e_{k 1}+o(t)\right)
$$

Разобьем множество $\{1, \ldots, m\}$ на подмножества $I_{1}, I_{2}, \ldots, I_{l}$, каждое из которых ссстоит из собственных чисел, равных между собой по абсолютной величине. Соответствующие абсолютные величины будем обозначать через $\lambda\left(I_{q}\right)$. Перенумеруем подмножества так, чтобы $\lambda\left(I_{1}\right)>\lambda\left(I_{2}\right)>\ldots>\lambda\left(I_{l}\right)$. Заметим, что одно из этих чисел равно $\left(\alpha^{*}\right)^{2}$, так как эта величина, в силу лемм 5 и 6 , есть собственное число оператора $G$, отвечающее собственному вектору $z^{*}=\left(x^{*}, a^{*} x^{*}\right)$. 
Запишем собственные числа $\lambda_{k}$ в тригонометрической форме: $\lambda_{k}=\left|\lambda_{k}\right|\left(\cos \varphi_{k}+\mathrm{i} \sin \varphi_{k}\right)$. Введем обозначения $v_{k \theta}^{\prime}=v_{k \theta} /\left|\lambda_{k}\right|^{\theta-1}(\theta-1) !$; $u_{k \theta}$ и $v_{k \theta}$ - векторы, составленные соответственно из реальных и мнимых частей координат вектора $e_{k \theta}$. Тогда (11) можно переписать в виде

$$
z_{t}=\sum_{q=1}^{l}\left(\lambda\left(I_{q}\right)\right)^{t} \sum_{k \in I_{q}} \sum_{\theta=1}^{\theta_{k}} t^{\theta-1} v_{k \theta}^{\prime}\left[u_{k 1} \cos (t-\theta+1) \varphi_{k}-\right.
$$

$\left.-v_{k 1} \sin (t-\theta+1) \varphi_{k}+\mathrm{i}\left(u_{k 1} \sin (t-\theta+1) \varphi_{k}+v_{k 1} \cos (t-\theta+1) \varphi_{k}\right)+o(t)\right]$.

Таким образом, решение $z_{t}$ предстает в виде суммы компонент, отвечающих различным значениям абсолютных величин и кратностей собственных чисел матрицы $G$. Поскольку координаты векторов $z_{t}$ заведомо вещественны, то мнимая часть в выражении (12) должна быть равна нулю**. Заметим, что величины $\cos (t-\theta+1) \varphi^{k}$ и векторы $u_{k \theta}$, отвечающие комплексным сопряженным собственным числам, совпадают, а соответствующие величины $\sin (t-\theta+1) \varphi_{k}$ и векторы $v_{k \theta}$ различаются лишь знаком. Следовательно, в (12) слагаемые, отвечающие комплексным сопряженным собственным числам, можно сгруппировать. Будем далее везде считать, что в выражении (12) каждая пара комплексных сопряженных собственных чисел представлена одним - тем, у которого мнимая часть положительна, так что $\varphi_{k} \in[0, \pi]$ для любого $k$.

В исследовании асимптотики выражения (12) существенную роль играет

Л е м м а 7. Пусть $\varphi_{k}$, где $k=1, \ldots, h$, - попарно различные числа из интервала $[0,2 \pi)$. Тогда для любого $\varepsilon>0$ найдется возрастающая последовательность натуральных чисел $\left\{\tau_{n}(\varepsilon)\right\}_{n=1}^{\infty}$ таких, что для всех $n=1,2, \ldots u k=1, \ldots, h$ выполняется

$$
-\varepsilon \leqslant \tau_{n} \varphi_{k}(\bmod 2 \pi)<\varepsilon,
$$

где $a(\bmod b)=a-[a / b] b$.

Доказательство этого утверждения основано на следующем факте теории динамических систем.

Л емма $8\left({ }^{5}\right]$, с. 68). Пусть преобразование $\simeq$ т-мерного тора $\operatorname{Tor}^{m}$ в себя задано формулой $\mathfrak{x}=\left(x_{1}+\alpha_{1}(\bmod 1), \ldots\right.$ $\left.\ldots, x_{m}+\alpha_{m}(\bmod 1)\right)$, где $x=\left(x_{1}, \ldots, x_{m}\right) \in \operatorname{Tor}^{m}, \quad$ причем числа $1, \alpha_{1}, \ldots, \alpha_{m}$ рационально независимы. Тогда траектория любой точки $x \in \operatorname{Tor}^{m}$, т. е. мнонество точек вида $\tau^{h} x$, всюду плотна на торе.

Если в задаче (6) $T>2 n$, то в силу лемм 3 и 4 вектор $z_{0}$ бесконечно продолжим, причем для соответствующей цепочки $\left\{z_{t}\right\}_{t=0}^{\infty}$ выполняется $z_{t}=G^{t} z_{0}$ и $z_{t} \in R_{\infty}$ для всех $t$. Исходя из этого, лемма 7 позволяет доказать, что составляющие

$$
\sum_{k \in I_{q}} \sum_{\theta=1}^{\theta_{k}} t^{\theta-1} v_{k \theta}^{\prime}\left(u_{k 1} \cos (t-\theta+1) \varphi_{k}-v_{k 1} \sin (t-\theta+1) \varphi_{k}\right)
$$

в выражении (12) также принадлежат множеству $R_{\infty}$ для любого $t \geqslant 0, q=1, \ldots, l$. Используя этот факт, лемму 5 , а также невырожденность матрицы вида $\quad\left(\cos \tau \varphi_{k}\right)_{\tau, k=0}^{h-1}, \quad$ где $\varphi_{k} \in[0, \pi]$

** Можно показать, что для этого необходимо, чтобы коэффициенты $v_{h_{\theta}}$, отвечающие комплексным сопряженным собственным числам, были попарно равны
между собой. 
попарно различны**;, можно получить следующее важное утверждение.

Л емм а 9. Собственные числа $\lambda_{k}$ матрицы $G$ для таких $k \in$ $\in\{1, \ldots, m\}$, что не все коэффициенты $v_{k \theta}$ в разложении (10) нулевые, яеляются и собственными числами пучка матриц (ㄱ, D), а соответствующие векторы $u_{k 1} u v_{k 1}$ принадлежат $R_{\infty}$.

Отсюда и из леммы 6(i) немедленно вытекает

Следствие. Пусть $\lambda_{k}$ - собственное число матрицы $G$ такое, что $\left|\lambda_{k}\right|>\left(\alpha^{*}\right)^{2}$ и коэффициенты $v_{k \theta}$, соответствующцие ему в разложении (10), не все равны нулю. Тогда число $\lambda_{k}$ либо комплексно (с ненулевой мнимой частью), либо отрицательно.

\section{3. Структура доказательства основного результата}

Обозначим через $Q_{1}$ множество индексов $q$, для которых $\left|\lambda\left(I_{q}\right)\right|>\left(\alpha^{*}\right)^{2}$, а через $\tilde{z}_{t}-$ составляющую разложения вектора $z_{t}$, лежащую в инвариантном подпространстве, определяемом собственными числами $\lambda_{k}$ такими, что $\left|\lambda_{k}\right|>\left(\alpha^{*}\right)^{2}$. Пусть $Q_{2}-$ множество индексов $q$, для которых $\left|\lambda\left(I_{q}\right)\right|<\left(\alpha^{*}\right)^{2}$, а $\hat{z_{t}}$ - соответствующая множеству $Q_{2}$ составляющая разложения вектора $z_{t}$.

Предложение 1. Для любого $\varepsilon>0$ найдется натуральное $T_{1}(\varepsilon)$ такое, что если выполняется $0 \leqslant t \leqslant[T / 2]-T_{1}(\varepsilon)$, то справедливо неравенство

$$
\left\|\tilde{z}_{t}\right\|<\frac{\varepsilon}{6}\left\|z_{t}\right\| .
$$

Схема доказательства. Фиксируем некоторый момент $t$ и рассмотрим разложение вектора $z_{t}$ по корневым векторам оператора $G$. Обозначим коэффициент разложения при векторе $e_{k \theta}$ через $v_{k \theta}(t)$ так, что

$$
z_{t}=\sum_{k=1}^{m} \sum_{\theta=1}^{\theta_{k}} v_{k \theta}(t) e_{k \theta} .
$$

Введем обозначения: $\theta(q)=\max _{k \in I_{q}} \theta_{k}, I_{q, \theta}=\left\{k \in I_{q} \mid \theta \leqslant \theta_{k}\right\}$.

Тогда можно записать

$$
\tilde{z}_{t}=\sum_{q \in Q_{1}} \sum_{\theta=1}^{\theta(q)} \sum_{k \in I_{q, \theta}} v_{k \theta}(t) e_{k \theta} .
$$

Заметим, что $z_{s}=G^{s-t} z_{t}$ при $t \leqslant s \leqslant[T / 2]-n-1$, следовательно, аналогично (12), при $t+T_{0} \leqslant s \leqslant[T / 2]-n-1$ имеем

$$
\begin{gathered}
\tilde{z}_{s}=\sum_{q \in \theta_{1}} \sum_{\theta=1}^{\theta(q)}\left(\lambda\left(I_{q}\right)\right)^{s-t}(s-t)^{\theta-1} \sum_{k \in I_{q, \theta}} v_{k \theta}^{\prime}(t)\left[u_{k 1} \cos (s-t-\theta+1) \varphi_{k}-\right. \\
\left.-v_{k 1} \sin (s-t-\theta+1) \varphi_{k}+o(s-t)\right],
\end{gathered}
$$

где $v_{k \theta}^{\prime}(t)=v_{k \theta}(t) /\left|\lambda_{k}\right|^{\theta-1}(\theta-1)$ !.

Внделим в разложении (14) компоненту $\tilde{z}_{t}\left(I_{1}, \theta(1)\right)=$ $=\sum_{k \in I_{1, \theta(1)}} v_{k \theta(1)}(t) e_{k \theta(1)}$. Напомним, что $I_{1}$ - подмножество собственных чисел оператора $G$, имеющих максимальный модуль, а $\theta(1)$ - наибольшая кратность, которую имеют собственные числа из $I_{1}$. Таким образом, в сумме (15) слагаемое

*** Нетрудно показать, что определитель этой матрицы равен константе, умноженной на определитель Вандермонда. 


$$
\begin{gathered}
\tilde{z}_{s}\left(I_{1, \theta(1)}\right)=\left(\lambda\left(I_{1}\right)\right)^{s-t}(s-t)^{\theta(1)-1} \sum_{k \in I_{1, \theta(1)}} v_{k \theta(1)}^{\prime}(t)\left[u_{k 1} \cos (s-t-\theta(1)+1) \varphi_{k}-\right. \\
\left.-v_{k 1} \sin (s-t-\theta(1)+1) \varphi_{k}+o(s-t)\right],
\end{gathered}
$$

отвечающее компоненте $\tilde{z}_{t}\left(I_{1, \theta(1)}\right)$, имеет наибольш̈ий порядок (оно содержит экспоненту с наибольшим основанием и полином наивысшей степени). Аналогичным образом опредделим в разложении (14) компооненты $\tilde{z}_{t}\left(I_{q, \theta}\right)$ для всех $\theta=1, \ldots, \theta(\dot{q}) ; q \in Q_{1}$. Чйло та丿ких ќомпонент $K=\sum_{q \in Q_{1}} \theta(q)$.

Докажем, что для компоненты нанбольшего порядка $\tilde{z}_{t}\left(I_{1, \theta(1)}\right)$ справедливо утверждение: для любого $\varepsilon>0$ найдется натуральное $\hat{N}_{1}=\hat{N}(\varepsilon, 1, \theta(1))$ такое, что если $0 \leqslant t \leqslant[T / 2]-N_{1}$, то имеет место

$$
\left\|\tilde{z}_{t}\left(I_{1, \theta(1)}\right)\right\|<\varepsilon\left\|z_{t}\right\| / 6 K .
$$

Для этого используется следующее вспомогательное утверждение, доказательство которого, опирающееся на лемму 7 , здесь не приводится.

Положим $\quad w_{s}=\sum_{k \in I_{1, \theta(1)}} v_{k \theta(1)}^{\prime}(t)\left[\dot{u}_{k 1} \cos (s-t-\theta(1)+1) \varphi_{k}-\right.$ $\left.-v_{k 1} \sin (s-t-\theta(1)+1) \varphi_{k}\right]$, так что $\tilde{z}_{s}\left(I_{1, \theta(1)}\right)=\left(\lambda\left(I_{1}\right)\right)^{s-t}(s-t)^{\theta(1)-1} \times$ $\chi\left(w_{s}+o(s-t)\right)$. Пусть для $\delta>0$ число $\tau(\delta)$ - минимальное натуральное из леммы 7 , т. е. такое, что $\left|\tau(\delta) \varphi_{k}(\bmod 2 \pi)\right|<\delta$ для $k=$ $=1, \ldots, m$.

Л е м м а 10. Сущзествует $\delta_{0}>0$ и константа $c_{1}>0$ такие, что для всякого $\delta \in\left(0, \delta_{0}\right)$, если $[T / 2]>t+T_{0}+\tau(\delta)$, то найдутся момент $s_{\delta} \in\{[T / 2]-\tau(\delta), \ldots,[T / 2]-1\} \quad$ и индекс $j \in\{1, \ldots, 2 n\}$ такие, чTO

$$
w_{s_{0}}^{(j)}<-\frac{c_{1}}{\tau(\delta)}\left\|\tilde{z}_{t}\left(I_{1, \theta(1)}\right)\right\|
$$

Доказательство леммы 10 , опирающееся на лемму 7 и следствие из леммы 9, здесь не приводится. Основной ее смысл состоит в том, что координаты составляющих величины́ вида $z_{r}=G^{r} z$, соответствующих наборам комплексных либо отрицательных собственных чисел, при достаточно больших $r$ с определенной периодичностью принимают отрицательные значения.

Будем доказывать (16) от противного, т. е. предполагая, что существует такое $\varepsilon>0$, что для сколь угодно большого $N$ найдутся $T>2 N$ и $t \in\{0, \ldots,[T / 2]-N\}$ такие, что

$$
\left\|\tilde{z}_{t}\left(I_{1, \theta(1)}\right)\right\|>\varepsilon\left\|z_{t}\right\| / 6 K .
$$

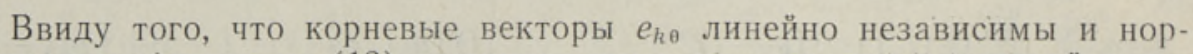
мированы, исходя из (13), нетрудно доказать, что для некоторой положительной константы $b$ справедлива оценка

$$
\left\|z_{t}\right\| \geqslant b \sum_{k=1}^{m} \sum_{\theta=1}^{\theta_{k}}\left|v_{k \theta}(t)\right|\left\|u_{k 1}\right\| .
$$

Поскольку $\quad \tilde{z}_{s}\left(I_{1, \theta(1)}\right)=\left(\lambda\left(I_{1}\right)\right)^{s-t}(s-t)^{\theta(1)-1}\left(w_{s}+o(s-t)\right)$, то пेрименяя лемму 10 , можно утверждать, что при достаточно больших $T\left([T / 2] \geqslant t+T_{0}+\tau(\delta)\right)$ справедливо неравенство

$$
\tilde{z}_{s_{\delta}}^{(j)}\left(I_{1, \theta(1)}\right)<\frac{-c_{1}}{2 \tau(\delta)}\left(\lambda\left(I_{1}\right)\right)^{s_{\delta}-t}\left(s_{\delta}-t\right)^{\theta(1)-1}\left\|\tilde{z}_{t}\left(I_{1, \theta(1)}\right)\right\|,
$$

что вместе с (17) дает 


$$
\tilde{z}_{s_{\delta}}^{(j)}\left(I_{1, \theta(1)}\right)<-\frac{c_{1} \varepsilon}{12 K \tau(\delta)}\left(\lambda\left(I_{1}\right)\right)^{s_{\delta}-t}\left(s_{\delta}-t\right)^{\theta(1)-1}\left\|z_{t}\right\| .
$$

Заметим, что по условию для всех $s \geqslant 0$ должно выполняться $z_{s} \geqslant 0$, тем самым и $z_{s_{0}}^{(j)} \geqslant 0$. Отсюда и из отрицательности $\tilde{z}_{s_{0}}^{(j)}\left(I_{1, \theta(1)}\right)$ следует

$$
\left|\tilde{z}_{s_{\delta}}^{(j)}\left(I_{1, \theta(1)}\right)\right|<z_{s_{\delta}}^{(j)}-\tilde{z}_{s_{\delta}}^{(j)}\left(I_{1, \theta(1)}\right) .
$$

Оценим сверху правую, часть неравенства (20). Она представляет собой сумму компонент (как в разложении (15)) меньшего порядка, чем $\tilde{z}_{s_{\delta}(j)}\left(I_{1, \theta(1)}\right)$, Точнее, при $[T / 2] \geqslant t+T_{0}+\tau(\delta)$ имеет место

$$
\begin{gathered}
z_{s_{\delta}}^{(j)}-\tilde{z}_{s_{\delta}}^{(j)}\left(I_{1, \theta(1)}\right) \leqslant \\
\leqslant 2\left(\lambda\left(I_{1}\right)\right)^{s_{0}-t}\left(s_{\phi}-t\right)^{\theta(1)-2} \sum_{k=1}^{m} \sum_{\theta=1}^{\theta_{k}}\left|v_{k \theta}^{\prime}(t)\right| \cdot \| u_{k 1} \cos \left(s_{\delta}-t-\theta+1\right) \varphi_{k}- \\
-v_{k 1} \sin \left(s_{\delta}-t-\theta+1\right) \varphi_{k} \| \leqslant \\
\leqslant 2 d\left(\lambda\left(I_{1}\right)\right)^{s_{\delta}-t}\left(s_{\delta}-t\right)^{\theta(1)-2} \sum_{k=1}^{m} \sum_{\theta=1}^{\theta_{k}}\left|v_{k \theta}(t)\right| \cdot\left\|u_{k 1}\right\|,
\end{gathered}
$$

где $d=1+\max _{k=1, \ldots, m}\left\|v_{k 1}\right\| /\left\|u_{k 1}\right\|, \quad$ поскольку, очевидно, $\quad\left|v_{k \theta}^{\prime}(t)\right| \leqslant$ $\leqslant\left|v_{k \theta}(t)\right|$. Сопоставляя эту цепочку неравенств с (18) и $(20)$, получим

$$
\left|\tilde{z}_{s_{0}}^{(j)}\left(I_{1, \theta(1)}\right)\right|<\frac{2 d}{b}\left(\lambda\left(I_{1}\right)\right)^{s_{0}-t}\left(s_{\delta}-t\right)^{\theta(1)-2}\left\|z_{t}\right\| .
$$

Отсюда и из (19) имеем $s_{\delta}-t<24 K \tau(\delta) d / b c_{1} \varepsilon$. очевидно, нарушается если $0 \leqslant t \leqslant[T / 2]-N$ при $N>N_{1}=$ $=24 K \tau(\delta) d / b c_{1} \varepsilon+\tau(\delta)$ и $[T / 2] \geqslant t+T_{0}+\tau(\delta)$.

Полученное противоречие завершает доказатељьство (16).

Исходя из этого, идентичным образом можно получить оценку вида (16) для следующей по порядку компоненты в разложении (14)$\tilde{z}_{t}\left(I_{1, \theta(1)-1}\right)-$ при $t \in\left\{0, \ldots,[T / 2\}-N_{1}-N_{2}\right\}$, ғде $N_{2}=N(\varepsilon, 1, \theta(1)-1)$, а затем последовательно для всех $K$ компоне:тт разложения (14) при $t=\left\{0, \ldots,[T / 2]-\sum_{i=1}^{K} N_{i}\right\} . \quad$ Тогда, применяя неравенство треугольника, можно получить требуемое неравенство $\left\|\tilde{z}_{t}\right\|<\varepsilon\left\|z_{t}\right\| / 6$, и для завершения доказательства предложения 1 остается положить $T_{1}(\varepsilon)=\max \left\{\sum_{i=1}^{K} N_{i}, T_{0}+\tau(\delta)\right\}$.

Аналогичный факт устанавливается для составляющей $\hat{z_{t}}$ разложения вектора $z_{t}$.

Предложение 2 . Для любого $\varepsilon>0$ найдется натуральное $T_{2}(\varepsilon)$ такое, ито если выполняется $T_{2}(\varepsilon) \leqslant t \leqslant[T / 2]-n-1 ;$ то справедливо неравенство

$$
\left\|\hat{z_{t}}\right\|<\frac{\varepsilon}{6}\left\|z_{t}\right\| .
$$

В его доказательстве используется тот факт, что $\left|\lambda\left(I_{q}\right)\right|<$ $<\left(\alpha^{*}\right)^{2}$ для $q \in Q_{2}$, а также легко получаемое соотношение для решений системы (6): $p^{*} \hat{B} x_{t}^{\prime}=\left(\alpha^{*}\right)^{t-1} p^{*} \hat{B} x_{1}^{\prime}+\frac{1}{\alpha^{*}} p^{*} \hat{D} x_{t-1}^{\prime}>$ 
$>\left(\alpha^{*}\right)^{t-1} p^{*} \hat{B} x_{1}^{\prime}$, где $p^{*}-$ левый вектор Фробениуса для матрицы $\alpha^{*} \hat{B}+\frac{1}{\alpha^{*}} \hat{D}$, т. е. $p^{*}=p^{*}\left(\alpha^{*} \hat{B}+\frac{1}{\alpha^{*}} \hat{D}\right)$.

Сопоставляя результаты предложений 1 и 2 , можно записать

$$
\left\|\tilde{z}_{t}+\hat{z}_{t}\right\|<\frac{\varepsilon}{3}\left\|z_{t}\right\|
$$

при $T_{2}(\varepsilon) \leqslant t \leqslant[T / 2]-T_{1}^{\prime}(\varepsilon)$, где $T_{1}^{\prime}(\varepsilon)=\max \left\{T_{1}(\varepsilon), n+1\right\}$. С другой стороны, из леммы 6, леммы 9 и разложения (11) следует, что

$$
\left\|\frac{z_{t}-\tilde{z}_{t}-\hat{z}_{t}}{\left\|z_{t}-\tilde{z}_{t}-\hat{z}_{t}\right\|}-\frac{z^{*}}{\left\|z^{*}\right\|}\right\|<\varepsilon
$$

когда $t$ не меньше некоторого $T_{3}(\varepsilon)$.

Используя свойства нормы, нетрудно показать, что при достаточно малых $\varepsilon>0$ из неравенства (21) следует при тех же значениях $t$, что вместе с (22) дает

$$
\left\|\frac{z_{t}}{\left\|z_{t}\right\|}-\frac{z_{t}-\tilde{z}_{t}-\hat{z}_{t}}{\left\|z_{t}-\tilde{z}_{t}-\hat{z}_{t}\right\|}\right\|<\varepsilon
$$

$$
\left\|\frac{z_{t}}{\left\|z_{t}\right\|}-\frac{z^{*}}{\left\|z^{*}\right\|}\right\|<2 \varepsilon
$$

при $\max \left\{T_{2}(\varepsilon), T_{3}(\varepsilon)\right\} \leqslant t \leqslant[T / 2]-T_{1}^{\prime}(\varepsilon)$. Напомним, что $z_{t}=$ $=\left(x_{2 t+1}^{\prime}, x_{2 t+2}^{\prime}\right), z^{*}=\left(x^{*}, \alpha^{*} x^{*}\right)$. Из (23) нетрудно вывести, что найдется константа $\hat{c}>0$ такая, что справедливы неравенства

$$
\left\|\frac{x_{2 t+1}^{\prime}}{\left\|x_{2 t+1}^{\prime}\right\|}-x^{*}\right\|<\hat{c} \varepsilon ; \quad\left\|\frac{x_{2 t+2}^{\prime}}{\left\|x_{2 t+2}^{\prime}\right\|}-x^{*}\right\|<\hat{c} \varepsilon ; \quad\left|\frac{\left\|x_{2 t+2}^{\prime}\right\|}{\left\|x_{2 t+1}^{\prime}\right\|}-\alpha^{*}\right|<\hat{c} \varepsilon
$$

при $\max \left\{T_{2}(\varepsilon), T_{3}(\varepsilon)\right\} \leqslant t \leqslant[T / 2]-T_{1}^{\prime}(\varepsilon)$. Таким образом, первая часть утверждения теоремы доказана, достаточно положить $T(\varepsilon)=2 \max \left\{T_{1}^{\prime}(\varepsilon / \hat{c}), T_{2}(\varepsilon / \hat{c}), T_{3}(\varepsilon / \hat{c})\right\}+1$.

Доказательство второй части утверждения теоремы, касающейся составляющей компенсации $\left\{x_{t}^{\prime \prime}\right\}$ проводится совершенно аналогично, применительно к системе (7), с той разницей, что вместо пучка матриц $(\mathfrak{B}, \mathfrak{D})$ рассматривается пучок $(\mathfrak{D}, \mathfrak{B})$, т. е. счет времени ведется как бы в противоположном направлении. При этом аналогично лемме 6 максимальным среди вещественных собственных чисел пучка (D, $\mathfrak{B})$ оказывается число $1 /\left(\alpha_{*}\right)^{2}$, и ему соответствует собственный вектор $z_{\star}=\left(x_{*}, \alpha_{\star} x_{*}\right)$.

\section{Л ИТ Е Р А Т Р А}

1. Каганович И. // Изв. АН ЭССР. Обществ. науки, 1983, 32, № 4, 277-289.

2. Мовшович C. М. // Экономика и математические методы, 1972, 8, № 2, $256-265$.

3. Аиманов C. А. // ЖВМ и МФ, 1983, 23, № 5, 1052-1059.

4. Гантмахер Ф. Р. Теория матриц. М., Наука, 1967.

5. Корнфельд И. П., Синай Я. Г. Фомин С. В. Эргодическая теория. М., Наука, 1980.

Ннститут кибернетики

Академии наук Эстонской ССР
Поступила в редакцию 29/IX 1988 


\section{KAGANOVITS}

\section{MAJANDUSE DUNAAMIKA JÄAKKKAHJUSID KOMPENSEERIVAS LEONTIEFI MUDELIS}

On uuritud Leontiefi dünaamilist mudelit, kus toodangu ja kulude bilansis on arvestatud ka jääkkahjude kompenseerimise kulud, ning näidatud, et sellises mudelis eksisteerivad statsionaarse kasvu maksimaalse ja ka minimaalse tempoga trajektoorid. On tõestatud, et vaadeldud mudeli alusel formuleeritud dünaamilise planeerimisülesande lahendeid saab aproksimeerida ülalmainitud kahe statsionaarse kasvu trajektoori teatud kombinatsiooni abil.

\section{KAGANOVICH}

\section{ECONOMIC GROWTH IN A LEONTIEF MODEL INCLUDING COMPENSATION OF RESIDUAL DAMAGES}

A Leontief-type dynamic model is considered, where the input-output balance includes in addition to accumulation ( f uture inputs»), also the costs caused by the activity in the previous time period (in particular, the compensation of damages) «past costs», The corresponding problem of the determination of the rate of balanced growth is proved to have both maximum and (in contrast to the standard models) non-zero minimum solutions. Thus, in this model, there exist optimal balanced paths corresponding to opposite goals: maximal growth of production and most rapid decrease of residual damages caused by previous activities.

The main result of the paper is a sort of turnpike theorem. For a finite-term planning problem based on the model in question, optimal programs are proved to be close to a certain combination of the above-mentioned balanced paths. Thus, optimal growth appears to be a result of the interaction of two components, responding to the processes of accumulation and compensation of residual damages. 